\title{
Min/Max Inventory Planning for Military Logistics
}

\author{
$\underline{\text { S. Shekh }}$ \\ Defence Science and Technology Organisation, Edinburgh, South Australia, Australia \\ Email:Slava.Shekh@dsto.defence.gov.au
}

\begin{abstract}
Military logistics is concerned with the projection and sustainment of forces in accomplishing a mission. A critical part of force sustainment is supply chain planning (SCP), which involves determining the sources of supply and defining the distribution networks that will be used to meet the sustainment requirements of the force. Although supply chain planning is well-researched in the commercial sector, the military are driven by different criteria and use different planning processes on which there is limited research.

In the military domain, supply chain planning involves determining the deliveries required to meet the expected demand of a particular mission by calculating suitable delivery times and supply quantities. This process is typically done using a system called Min/Max, which calculates the deliveries using minimum (Min) and maximum (Max) inventory levels and a number of other constraints. The basic idea is to resupply the inventory of a node (location) when the stock level approaches, but doesn't fall below, the Min inventory level and order enough supplies to bring the stock level up to the Max stock holding capacity. This is traditionally a manual process that can take a group of military planners several hours or days to complete, depending on the size of the problem, and the results are susceptible to human error.

This paper presents the Min/Max Planning Algorithm (MPA) which can perform automated supply chain planning using the Min/Max system. Given an SCP problem with an expected profile of demand, the MPA uses the constraints defined for each node and the expected demand to calculate a suitable order profile for that node. Each node's order profile is combined with the profiles of its dependants, providing a complete picture of the supplies that a node will require to sustain itself and its dependants over the course of the mission. These combined order profiles are then converted into a list of deliveries which provides an overall solution to the SCP problem.
\end{abstract}

The MPA has been evaluated using four SCP problems of increasing complexity, which are representative of typical planning problems encountered by the Australian Defence Force. The results show that the MPA is able to solve all of these problems and keep the expected minimum stock level above a defined threshold, which provides a suitable buffer against uncertainty when the calculated deliveries are actually executed. The algorithm is also computationally efficient, being able to solve large SCP problems in less than a second. This is a significant improvement on the hours or days that it could take a military planner to perform the same calculations by hand.

Keywords: Supply chain planning, inventory planning, min/max, military logistics 


\section{INTRODUCTION}

Logistics can be defined as "the detailed coordination of a complex operation involving many people, facilities, or supplies" (OxfordDictionaries, 2014). In the military domain, logistics is concerned with the projection of forces and the sustainment of those forces in accomplishing a task or mission (Tuttle, 2005). A critical part of force sustainment is determining the sources of supply and defining the distribution networks that will be used to meet the sustainment requirements of the force (Peltz, et al., 2008). This process is referred to as supply chain planning (SCP).

Although logistics and supply chain planning are central to both commercial and military operations, the key motivators are largely different. In business logistics, decision making is typically driven by the desire to maximise profit while reducing costs. During peacetime, Defence may also seek to reduce costs in its logistics processes, but during conflict or wartime, the focus moves towards prosecuting operational outcomes while reducing the likelihood of injury and loss of life (Kovacs \& Tatham, 2009). Consequently, Defence follows its own set of processes for logistics and supply chain planning that address its unique requirements. For example, there is a preference to maintain stock on hand to mitigate against risk, rather than completely relying on a just-in-time approach.

\section{MOTIVATION}

Despite the importance of logistics to military operations, limited research is being undertaken with some measures indicating a slight decline in recent years (Yoho, et al., 2013). One key area that has been identified as requiring further work is "managing the logistics supply network" (Yoho, et al., 2013, p. 10), which includes both inventory management and stock positioning. Although this is a well-researched area in the commercial sector, the military deal with a different supply chain planning problem which necessitates the need for Defence-specific research. A brief example of an SCP problem is given below, while a more complete formulation can be found in Section 4.

In the current planning process, Australian Defence Force (ADF) planners are often required to perform manual calculations. For example, given a profile of expected demand of different supply items across various nodes (representing physical locations) a planner may need to determine the most suitable supply networks to use. Using those networks, the planner then needs to calculate a series of suitable reorder points and quantities to supply the nodes and meet the expected demand. In a scenario with 10 nodes, 10 supply networks, and a 90-day demand period at each node, the planner may need to calculate 100-500 unique reorder points and quantities, depending on how regularly supplies are required.

This process could take a group of planners hours or days to calculate manually, and due to the sheer number of calculations, the process is susceptible to human error. This paper presents an algorithm that can perform the same calculations in the order of milliseconds while reducing the likelihood of human error. The algorithm is designed around the specific requirements of the ADF and has been successfully integrated into a military planning system.

\section{RELATED WORK}

The ADF typically conduct their inventory planning using a system called Min/Max. The basic idea is to resupply a node's inventory when the stock level approaches, but doesn't fall below, the Min inventory level and order enough supplies to bring the stock level up to the Max stock holding capacity. One of the earliest mathematical formulations of this type of inventory planning is given by Arrow et al. (1951), while variations and extensions are described by Silver et al. (1998). Although neither work explicitly refers to the process as Min/Max, the methods they describe are very similar to the Min/Max system used by the ADF, which is further explained in Section 5.

There are many examples where Min and Max levels have been incorporated into inventory planning in the commercial domain. In a work on the effects of information synchronization on supply chain stability, Min and Max were used to calculate dispatch quantities between a manufacturer and a retailer (Venkateswaran \& Son, 2005). Another work employed minimum and maximum inventory levels to determine effective operating policies at supply hubs (Shah \& Goh, 2006). Other works may also use similar concepts, but simply refer to them using different terminology (Silver, et al., 1998).

In the military domain, research into supply chain and inventory planning is more limited (Yoho, et al., 2013). Ghanmi et al. (2009) propose a framework for the design of military supply networks, while Bean (2011) explores inventory management in situations where the presence of uncertainty can have devastating consequences, with the military environment being a prime example. Although these works are relevant, the 
research proposed in this paper is concerned with a fundamentally different inventory problem. The author isn't aware of any work that applies Max/Min inventory planning to the ADF Supply Chain Planning problem, as will be described in Section 4, and hence this is the motivation for our work.

In the inventory and supply chain literature, Min/Max and Max/Min can also refer to an application of the Minimax Theorem (Neumann, 1928) to the inventory problem. This involves either minimizing the maximum loss or maximizing the minimum profit over a range of demand distributions, thereby preparing for the worst case (Scarf, 1958). This shouldn't be confused with the Min/Max system described in this paper, which is an inventory planning system that helps maintain sufficient stock holdings throughout a military operation.

\section{PROBLEM DEFINITION}

In order to develop an algorithm for the ADF Supply Chain Planning (SCP) problem, we have created a model to represent the actual problem as accurately as possible. In our model, an SCP problem consists of an expected demand profile, two or more nodes, and one or more supply networks. The goal is to meet the expected demand within the constraints of the defined nodes and networks by ordering suitable quantities of supply items at appropriate times. An example of a simple SCP problem is shown in Figure 1 and the problem is further explained below.

Network 1 (Item X)

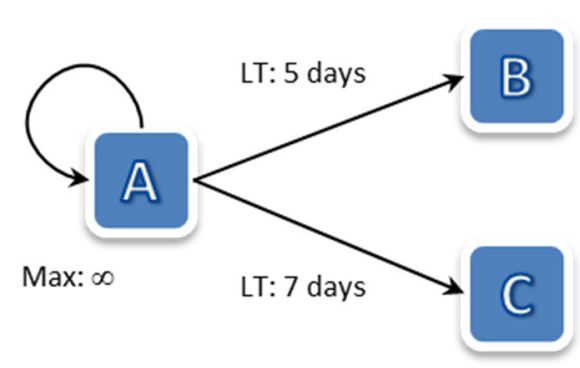

Max: $130 \mathrm{~m}^{3}$

Min: 4 DOS

CR: 3 DOS

IS: $50 \mathrm{~m}^{3}$

Max: 30 DOS

Min: 5 DOS

CR: 3 DOS

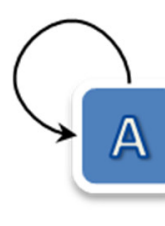

Max: $\infty$
Network 2 (Item Y, Item Z)

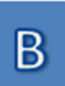

Max: 100 tonnes
Min: 20 tonnes CR: 3 DOS

\section{Expected Demand Profile}

Node B

Network 1

Day 0-Day 30

$10 \mathrm{~m}^{3}$ of Item $X$ per day

Node C

Network 1

Day 0-Day 10

$10 \mathrm{~m}^{3}$ of Item $X$ per day

Network 2

Day 10 - Day 20

4 tonnes of Item $Y$ per day

Day 20-Day 30

4 tonnes of Item $Y$ per day

2 tonnes of Item Z per day

Figure 1. An example SCP problem.

A supply network can be defined for a specific supply item or for a Class of Supply (COS), which represents a collection of supply items. To simplify the calculations, we assume that a node can only have 1 supplier per network, but it can supply multiple dependent nodes. A node can potentially be its own supplier, in which case it is referred to as a self-sustaining node and is assumed to be able to supply itself indefinitely with supply items associated with that network. In the ADF context, this is generally used to model the Australian National Support Base (NSB). The notion of having multiple nodes and networks in our problem is similar to multi-installation and multi-echelon inventory planning in the literature (Clark \& Scarf, 1960).

For each supply network, a node has a Max that defines the node's stock holding capacity, a Min that defines the minimum allowed inventory level, and a Commander's Reserve (CR) that acts as a buffer for both Min and Max. These node properties are defined by the planner based on the requirements and nature of the mission. The properties can be specified in units of volume, units of weight or days of supply (DOS). A single DOS is equivalent to the total demand quantity on one particular day, so the exact physical amount represented by a DOS can vary over time. In addition, a node may have initial stocks (IS) which can be used to reduce the total amount of supplies that need to be ordered through the supply chain. 
The links in each supply network have associated lead times (LT), defining the worst-case expected time for moving supplies along that link, including any time required for loading and unloading. This is typically an estimate made by the planner based on the transports that are expected to service the link.

The expected demand profile is calculated based on projected consumption rates of force elements taking part in the mission. The profile is stored in a hierarchical structure with the specific demand quantities of each supply item forming the base of the structure. These demand quantities can either be uniform across the whole mission or variable over time in which case they are subdivided into multiple time periods of uniform demand. These periods of demand are associated with particular supply networks, and the networks are associated with particular nodes, which form the top level of the structure. The total demand for a node will be referred to as node demand, the total demand for a network as network demand etc. For instance, the network demand for Network 2 in Figure 1 is 80 tonnes of Item Y (4 tonnes per day for 20 days, i.e. from day 10 to day 30 ) and 20 tonnes of Item Z ( 2 tonnes per day for 10 days).

Military operations are often characterised by uncertainty (Bean, 2011) so the actual demand during the mission can be very different to the forecast demand. However, rather than modelling demand stochastically to cater for variability, the military planning process involves using the Min, Commander's Reserve and lead time parameters to create a buffer against uncertainty. Lead times are often given higher values to allow for variability in delivery times, Min is typically set to a quantity representing expected consumption over the lead time, while Commander's Reserve is a form of reserve stock which is used as a further buffer. During the mission, if stocks fall below the planned Min level, the Min acts as a buffer to prevent stockout. Even if stocks fall well below Min, the Commander's Reserve is there to provide a further safeguard.

\section{MIN/MAX PLANNING ALGORITHM}

Finding a solution to an SCP problem by hand can be a very time consuming and error-prone process (see the example in Section 2). The Min/Max Planning Algorithm (MPA) has been developed to perform the underlying calculations and help automate this process. The MPA builds on the Supply Shaping Algorithm (SSA), which is a predecessor to the MPA and addresses the same kind of problem using a different approach. Rather than using Min, Max and Commander's Reserve, the SSA aims to keep inventory levels at a certain number of days of supply by resupplying each node at regular intervals. Although the SSA provides a solution to the problem, the MPA is more closely based on the ADF approach and therefore provides a more accurate model of the planning processes used by military planners.

The MPA works by constructing an order profile for each node based on the expected demand at that node within each network. The stock level for each node/network pair begins at zero and the demand day begins at the start time of the first time period. For simplicity, Max, Min and Commander's Reserve are automatically converted into the same units, and Commander's Reserve is added to both Min and Max to provide a buffer.

Based on the demand of each supply item in each time period associated with the node/network pair, orders are placed on the demand day to try to bring the stock level up to Max. Once Max is reached, the full demand from each time period is applied in succession until the stock level reaches or falls below Min. The point at which it was reached is then calculated and stored as the new demand day. This process is repeated until all supply items, time periods, networks and nodes have been considered, which results in a complete order profile for each node. As an example, Figure 2 provides a visualisation of the calculated order profile for Node B in Network 1 based on the SCP problem in Figure 1.

Once all order profiles have been calculated, the order profile of each node is aggregated with the profiles of its dependants, combining the order quantities when orders occur on the same day. A node $\mathrm{Y}$ is dependent on a node $\mathrm{X}$, if node $\mathrm{X}$ is directly or indirectly responsible for supplying node $\mathrm{Y}$. In Network 2 of Figure 1, C is a dependant of $\mathrm{B}$, and both $\mathrm{B}$ and $\mathrm{C}$ are dependents of $\mathrm{A}$. The aggregated order profile for each node is then converted into a list of required deliveries. The start time of each delivery is set to the time it is required (the demand day) minus the total lead time between the source of the demand and the supplier (i.e. in Network 2 of Figure 1 the lead time between node $\mathrm{A}$ and $\mathrm{C}$ is 8 days). The required deliveries for each node are added to a combined list of deliveries, and the combined list provides a solution to the SCP problem.

Although simple Min/Max inventory planning can easily be done by human planners, the algorithm provides an effective way of solving the more complex problems. The MPA applies the Min/Max method across multiple nodes, networks, supply items and time periods to form a very detailed and intricate order profile, which becomes impractical and error prone to calculate by hand for large SCP problems. Particularly when a network includes many supply items, manually determining the exact order points for individual items can be very difficult and therefore becomes a suitable problem for the algorithm. 


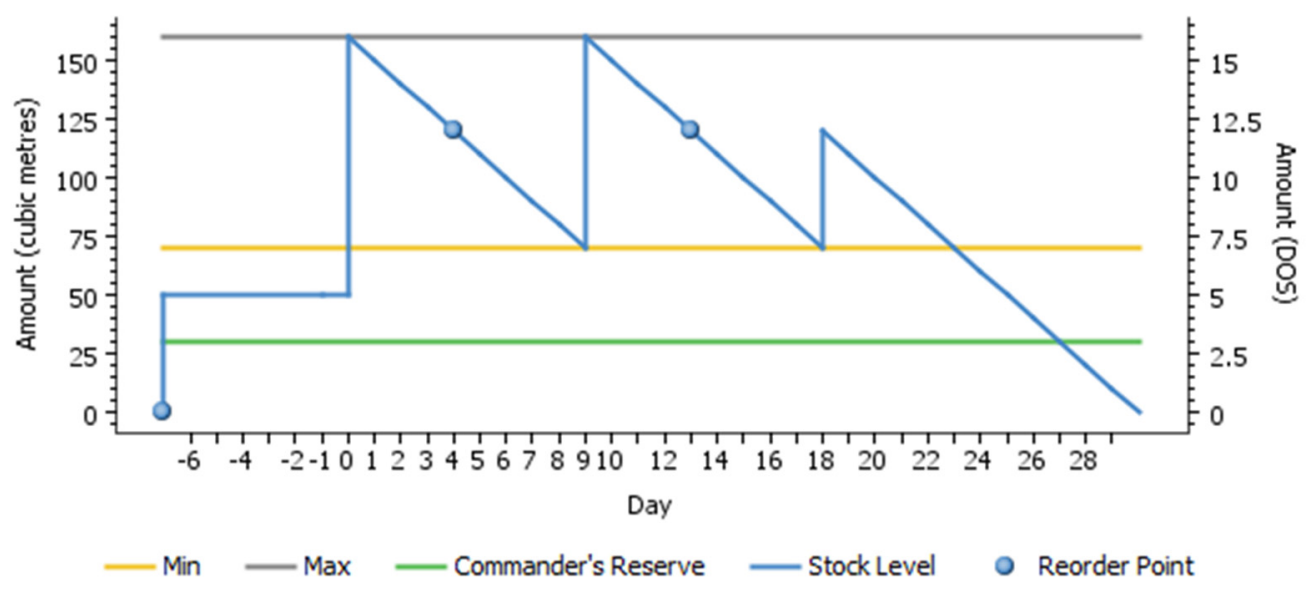

Figure 2. Calculated order profile for Node B in Network 1.

The Min, Max and Commander's Reserve of a node can also be specified in different units to each other, which can be in different units to the next node etc. Since units can be exact physical quantities, such as tonnes, or quantities that can vary daily depending on demand, such as DOS, the conversion between units and the calculation of the exact physical quantity required at a given time can be a challenging problem. The MPA automatically performs these conversions during calculation, ensuring that the correct stock level is maintained for the duration of the mission regardless of demand intensity.

\section{EVALUATION}

The MPA has been evaluated using four SCP problems of increasing complexity, which are representative of typical planning problems encountered by the ADF. Given a problem as input, the algorithm produces a list of required deliveries to satisfy the overall demand and an expected minimum stock level based on the Min values specified in the problem.

The MPA was developed in Java and run on a standard laptop with an Intel i7-2620M 2.70GHz CPU, 8 GB RAM and Windows 7 Enterprise x86-64. Table 1 provides an outline of each problem, the results produced by the algorithm and the computation time taken to calculate the deliveries. Although the computation times were only recorded for a single run, the algorithm and problems were completely deterministic so the results are repeatable. Rather than providing the complete expected demand profile for each problem, the total demand and demand duration specify the overall demand quantity and time period that is covered by the profile in each problem. Problem 1 is visualised in Figure 1.

Table 1. Results of running the MPA on four SCP problems of increasing complexity.

\begin{tabular}{|c|c|c|c|c|}
\hline & Problem 1 & Problem 2 & Problem 3 & Problem 4 \\
\hline Nodes & 3 & 6 & 10 & 15 \\
\hline Networks & 2 & 4 & 7 & 10 \\
\hline Supply items & 3 & 6 & 10 & 14 \\
\hline Total demand & $500 \mathrm{~m}^{3}$ & $5,300 \mathrm{~m}^{3}$ & $21,300 \mathrm{~m}^{3}$ & $102,150 \mathrm{~m}^{3}$ \\
\hline Demand duration & 30 days & 60 days & 150 days & 300 days \\
\hline Time periods & 4 & 9 & 17 & 27 \\
\hline \multicolumn{5}{|c|}{ Max/Min Planning Algorithm } \\
\hline Calculated deliveries & 12 & 34 & 124 & 405 \\
\hline Computation time & $32 \mathrm{~ms}$ & $50 \mathrm{~ms}$ & $85 \mathrm{~ms}$ & $115 \mathrm{~ms}$ \\
\hline Expected minimum stock & $25 \mathrm{~m}^{3}$ & $150 \mathrm{~m}^{3}$ & $250 \mathrm{~m}^{3}$ & $300 \mathrm{~m}^{3}$ \\
\hline
\end{tabular}

The results show that the MPA can effectively solve a range of SCP problems by keeping the minimum stock level above a specified Min threshold, which is always above zero and therefore avoids stockout. Given that Problem 4 represents the upper limit of an expected ADF SCP problem, the algorithm is also computationally efficient enough for our purposes, and significantly faster than producing a solution manually, which could potentially take hours or days. For example, given the input parameters of Problem 4, a planner would need to calculate 405 separate deliveries. Each delivery requires determining the delivery locations, timing, as well 
as the types and quantities of supply items being delivered. Using an optimistic estimate of 5 minutes per delivery calculation, it could take a planner around 34 hours to perform these calculations manually.

As well as providing an efficient method for solving SCP problems, the methodology used by the MPA is preferred by users when compared to alternate planning methods. Feedback received from various ADF users has indicated that the MPA approach is preferred because it closely aligns with the actual methods of supply chain planning used by the ADF.

Although the MPA doesn't consider transportation assets, lead times between nodes can be specified in each SCP problem. These lead times are typically estimates of expected delivery times between nodes based on the transports that are expected to be available. The MPA uses these lead times to determine how early to commence a delivery and therefore values should be set higher to cater for potential uncertainty during execution. Once the MPA has been run, the calculated deliveries can be tested for feasibility using an algorithm that solves the resource allocation problem, such as the one proposed by Allard \& Shekh (2012).

\section{CONCLUSIONS AND FUTURE WORK}

The Min/Max Planning Algorithm provides an automated way of solving ADF Supply Chain Planning problems. The algorithm attempts to model the actual planning methods used by the ADF, thereby trying to solve the problem by using similar techniques to those that would be used by a human planner. By using ADF planning methods, the solution produced is representative of an ADF solution and more likely to receive user acceptance. No other work in the literature has been found to address this specific problem.

The algorithm has been tested on many SCP problems, including the four problems mentioned in this paper, which are representative of typical ADF planning problems. The results show that the MPA is able to solve all of these problems and keep the expected minimum stock level above a defined threshold, which provides a suitable buffer against uncertainty when the calculated deliveries are actually executed. The algorithm is also computationally efficient, being able to solve large SCP problems in less than a second. This is a significant improvement on the hours or days that it could take a military planner to calculate the required deliveries for a large SCP problem, and their results would be subject to human error.

Future work could directly compare the solutions produced by the MPA to manual solutions produced by military planners. Differences between the calculated and manual solutions may reveal areas where the modelling of the problem and the algorithm could be further improved. For example, in the current model the lead time is represented as a single value per link, but in reality, a link may be serviced by multiple transports which would require multiple lead time values. Other future work could explore the use of stochastic demand profiles and lead times in the supply chain model to more closely align the algorithm with real-world military supply chain planning.

\section{ACKNOWLEDGEMENTS}

The author would like to acknowledge Luke Marsh for providing ideas and feedback throughout this project and Tony Allard for his key involvement in the development of the supply shaping algorithm (the predecessor of the Min/Max Planning Algorithm).

\section{REFERENCES}

Allard, T., \& Shekh, S. (2012). Hierarchical multi-agent distribution planning. In AI 2012: Advances in Artificial Intelligence (pp. 755-766). Springer.

Arrow, K. J., Harris, T., \& Marschak, J. (1951). Optimal inventory policy. Econometrica: Journal of the Econometric Society, 250-272.

Bean, W. L. (2011). Inventory management under uncertainty: a military application. University of Pretoria.

Clark, A. J., \& Scarf, H. (1960). Optimal policies for a multi-echelon inventory problem. Management Science, 6(4), 475-490.

Ghanmi, A., Martel, A., Berger, J., \& Boukhtouta, A. (2009). A framework for the design of a military operational supply network. In IEEE Symposium on Computational Intelligence for Security and Defense Applications (pp. 1-9). IEEE.

Kovacs, G., \& Tatham, P. (2009). Responding to Disruptions in the Supply Network: From Dormant to Action. Journal of Business Logistics, 30(2), 215-229. 
Shekh, S., Min/Max Inventory Planning for Military Logistics

Neumann, J. v. (1928). Zur theorie der gesellschaftsspiele. Mathematische Annalen, 100(1), 295-320.

OxfordDictionaries. (2014). logistics. Retrieved March 17, 2014, from http://www.oxforddictionaries.com/definition/american_english/logistics

Peltz, E., Girardini, K. J., Robbins, M., \& Boren, P. (2008). Effectively Sustaining Forces Overseas While Minimizing Supply Chain Costs: Targeted Theater Inventory. Rand Corporation.

Scarf, H. (1958). A min-max solution of an inventory problem. Studies in the mathematical theory of inventory and production, 10, 201-209.

Shah, J., \& Goh, M. (2006). Setting operating policies for supply hubs. International Journal of Production Economics, 100(2), 239-252.

Silver, E. A., Pyke, D. F., \& Peterson, R. (1998). Inventory management and production planning and scheduling. Wiley New York.

Tuttle, W. G. (2005). Defense logistics for the 21st century. Naval Institute Press.

Venkateswaran, J., \& Son, Y.-J. (2005). Information synchronization effects on the stability of collaborative supply chain. Proceedings of the 37th Conference on Winter Simulation, (pp. 1668-1676).

Yoho, K. D., Rietjens, S., \& Tatham, P. (2013). Defence logistics: an important research field in need of researchers. International Journal of Physical Distribution \& Logistics Management, 43(2), 80-96. 